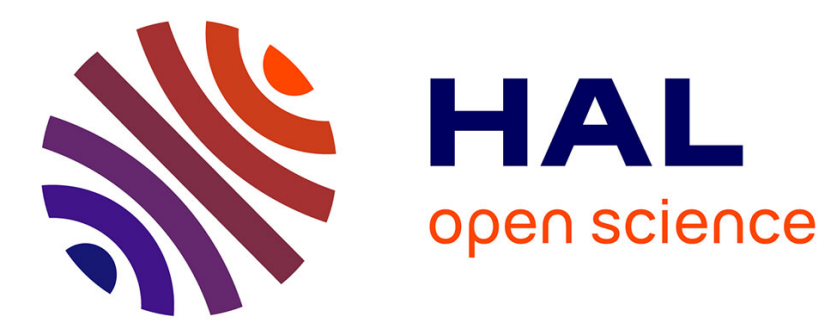

\title{
The integrals of small-angle scattering and their calculation \\ W. Gille
}

\section{To cite this version:}

W. Gille. The integrals of small-angle scattering and their calculation. Journal de Physique IV Proceedings, 1993, 03 (C8), pp.C8-503-C8-506. 10.1051/jp4:19938104 . jpa-00252234

\section{HAL Id: jpa-00252234 https://hal.science/jpa-00252234}

Submitted on 1 Jan 1993

HAL is a multi-disciplinary open access archive for the deposit and dissemination of scientific research documents, whether they are published or not. The documents may come from teaching and research institutions in France or abroad, or from public or private research centers.
L'archive ouverte pluridisciplinaire HAL, est destinée au dépôt et à la diffusion de documents scientifiques de niveau recherche, publiés ou non, émanant des établissements d'enseignement et de recherche français ou étrangers, des laboratoires publics ou privés. 


\title{
The integrals of small-angle scattering and their calculation
}

\author{
W. GILLE
}

Martin-Luther-Universität Halle-Wittenberg, FB Physik PF8, Kröllwitzer Str. 44, 06099 Halle, Germany

\begin{abstract}
This article explains a new concept concerning the integrals of SAS and their direct calculation from realistic experiments. Some new synonymous formulas for the calculation of the correlation function and its derivatives are represented. Some relations lead to a minimal truncation error and the error propagation can be investigated exactly. The fundamental idea for this is the "exchange" of the variables $r$ and $h$ with respect to differentiation, followed by an integration by parts. The result is not only one given formula. A table in the end allows the experimenter to select the representation he thinks to be the best one.
\end{abstract}

\section{Introduction}

It is often simply said that the structure functions of SAs can be calculated simply by Fourier transformation from the intensity I(h)

(pin-hole curve), respectively $\tilde{I}(h)$ (so-called infinitely smeared curve). $(\mathrm{h}=4 \cdot \pi \cdot \sin (\theta) / \lambda ; 2 \theta$-scattering angle; $\lambda$-wavelength).

The usual opinion leaves the problem to specialists of numerical mathematics. This is a very superficial view, because there are many methods and variants to determine integrals. These questions are dealt with in detail in the mathematical theory of not correctly formulated problems.

In 1955 GUINIER and FOURNET [3] published their book x-ray small-angle-scattering, in which they precisely and in detail showed the physical fundamentals leading to sAsintegrals. However, they did not deal with the calculation and transformation of the parameter integrals developed by them. It was 20 years later, when GLATTER [2] introduced his method, the indirect Fourier transformation, into sAs. There exist many other variants and methods.

In spite of this progress many scientists are of the opinion that derivations from structure functions $[4,1]$ are computed only by "very courageous" scientists. They rather concentrate on a direct comparison of experiment and model by $I(h)$-fitting.

There is no doubt that a numerical calculation of a structure function by points, for instance the correlation function $\gamma(r)$, followed by numerical differentiation in the second step, without taking into consideration truncation errors will hardly provide good results [1].

This is also right in the case of a nearly ideal experiment. Simple tests show that the knowledge of 400 recorded points $\left\{h_{k}, I_{k}\left(h_{k}\right)\right\}$, in the interval $0.1<\mathrm{h} \cdot \mathrm{D}<10$ does not give a stable intersect distribution function $\gamma^{\prime \prime}(r)$ without special tricks. 
2. Differentiation of a parameter integral $g(r)$ with respect to $r$

If an improper parameter integral $g(r)$ converges uniformly with respect to a parameter $r$, it can be differentiated and integrated according to this parameter. The resulting integral can be further integrated.

$$
g(r)=\int_{0}^{\infty} I(h) \cdot f(h, r) d h \quad, \quad \frac{\partial g(r)}{\partial r}=\int_{0}^{\infty} I(h) \cdot \frac{\partial f(h, r)}{\partial r} d h \quad 0 \leq r<\infty
$$

According to this strategy the second derivative of the correlation function $\gamma^{\prime \prime}(r)$ is obtained $[4,1]$ :

$$
\begin{aligned}
& \gamma^{\prime \prime}(r)=-\int_{0}^{\infty} I(h) h^{3} \frac{\sin (h r)}{r} d h-2 \int_{0}^{\infty} I(h) h^{2} \frac{\cos (h r)}{r^{2}} d h+2 \int_{0}^{\infty} I(h) h \frac{\sin (h r)}{r^{3}} d h \\
& \text { Normalisation is possible with } \gamma(0)=\int_{0}^{\infty} I(h) \cdot h^{2} d h=1 .
\end{aligned}
$$

Sometimes this method has a decisive disadvantage: The error propagation from I(h) to $\gamma^{\prime \prime}(r)$ cannot be easily investigated. On the other hand the following relation for the calculation of the so called interface distribution function $g(r),[1]$, which is used for the determination of size distribution of layers, for instance, in polymer physics.

$$
g(r)=\frac{1}{2} \int_{0}^{\infty} h^{4} \cdot I(h) \cdot \cos (h \cdot r) d h \equiv \frac{1}{2} \int_{0}^{\infty}\left[h^{4} \cdot I(h)\right]^{\prime} \cdot \frac{\sin (h \cdot r)}{r} d h
$$

The idea of this formula can be obviously continued [1].

\section{Some other useful representations from integration by parts}

A new type of transformations, which has not been investigated yet, appears, if we try to "exchange" the differentiation with respect to parameter $h$ into a differentiation with respect to the variable $r$.

$$
\gamma^{\prime \prime}(r)=+\frac{1}{I^{3}} \cdot \int_{0}^{\infty}\left[9 h^{2} \cdot I(h)+7 h^{3} \cdot I^{\prime}(h)+h^{4} \cdot I^{\prime \prime}(h)\right] \cdot \frac{\sin (h \cdot x)}{h} d h \text {. }
$$

In this formula the symbol ' is the derivative according to the argument that means according to $h$ in the integral. Another form of this is:

$$
\frac{\partial^{2} \gamma(r)}{\partial x^{2}}=\frac{\partial^{2}}{\partial r^{2}} \int_{0}^{\infty} h^{2} I(h) \cdot \frac{\sin (h x)}{h r} d h \equiv \frac{1}{r^{3}} \int_{0}^{\infty}\left[h^{4} \cdot I(h)\right] " \frac{\sin (h r)}{h} d h
$$

It looks strange, but it is correct indeed and will be explained now.

If the parameter integral for the correlation function in the first step is once partially integrated with respect to the variable $r$, we obtain the intermediate step:

$$
\gamma^{\prime}(r)=\frac{\partial \gamma(r)}{\partial r}=\int_{0}^{\infty} h^{2} I(h) \cdot \frac{\partial}{\partial x}\left(\frac{\sin (h \cdot r)}{h \cdot r}\right) d h
$$

As in the integrand there is now the derivative of a function, further integration by parts is possible. For this step we use the fact that the variables $h$ and $r$ occur "symmetrically". Obviously holds :

$$
\frac{\partial}{\partial r} \frac{\sin (h \cdot r)}{h \cdot r} \equiv \frac{h}{r} \cdot \frac{\partial}{\partial h} \frac{\sin (h \cdot r)}{h \cdot r} \text {. }
$$

So follows the line of partial integration:

$$
r \gamma^{\prime}(r)=\lim _{h \rightarrow \infty}\left[h^{3} I(h) \frac{\sin (h r)}{h r}\right]-\lim _{h \rightarrow 0}\left[h^{3} I(h) \frac{\sin (h r)}{h r}\right]-\int_{0}^{\infty}\left[h^{3} I(h)\right] \cdot \frac{\sin (h r)}{h r} d h \quad .
$$

Applying POROD's law holds: 


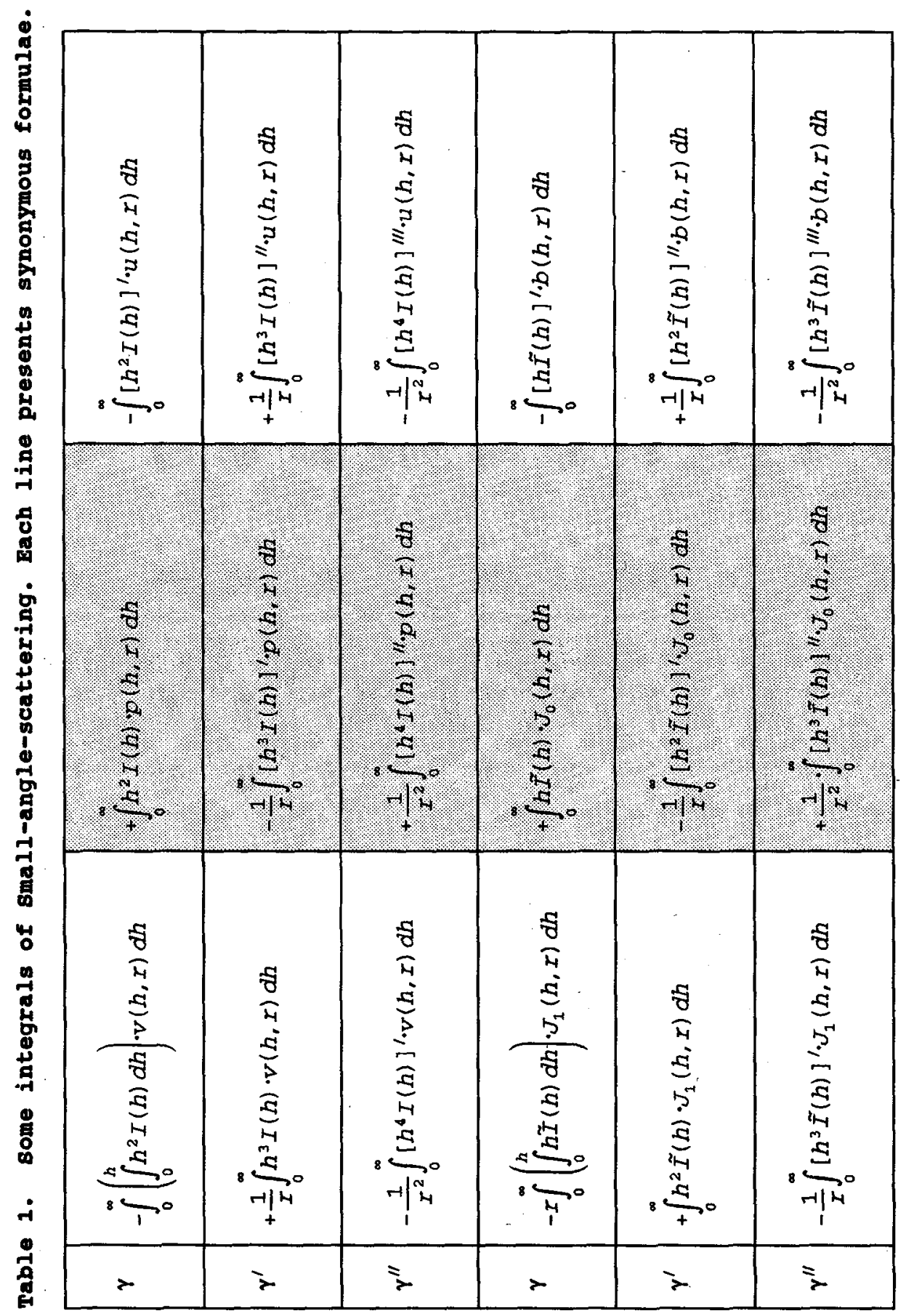




$$
\gamma^{\prime}(r)=-\frac{1}{r} \cdot \int_{0}^{\infty}\left[h^{3} \cdot I(h)\right]^{\prime} \cdot \frac{\sin (h r)}{h r} d h \quad \frac{\lim _{h \rightarrow \infty}\left[h^{3} \cdot I(h)\right]^{\prime}}{\lim _{h \rightarrow \infty}\left[h^{3} \cdot I(h)\right]}=0 .
$$

In this relation a partial derivative with respect to $r$ outside the integral is connected with one with respect to $h$ under the integral.

In some cases the truncation error can be reduced, putting on with the error occurring in the differentiation $I^{\prime}(h)$. An optimal integral representation depends on the type (noise, behavior for large $h$, limits of $h-$ interval $h_{\min }<h<h_{\max }$ ) of the recorded scattering function [1]. In table 1. the integrals in the hatched squares seem to be the most favorable [1]. In order to achieve the second derivative, the intermediate result for the first derivative is partially differentiated with respect to $r$ as done in the first step:

$$
r \cdot\left[r \gamma^{\prime}(r)\right]=-\int_{0}^{\infty}\left[h^{3} \cdot I(h)\right]^{\prime} \cdot h \cdot \frac{\partial}{\partial h} \frac{\sin (h r)}{h I} d h,
$$

Further integration by parts yields then:

$$
r \cdot\left[r \gamma^{\prime}(r)\right]^{\prime}=-\left[\left(h^{3} I(h)\right)^{\prime} \cdot \frac{\sin (h r)}{r}\right]_{0}^{\infty}+\int_{0}^{\infty}\left[\left(h^{3} I(h)\right)^{\prime} \cdot h\right]^{\prime} \cdot \frac{\sin (h r)}{h r} d h
$$

The two limiting values, contained in the first term of the relation above, will be calculated in the following way:

The first integration limit $h \rightarrow 0$ is not complicated. The limit is obviously zero.

The limit $\mathrm{h} \rightarrow \infty$ gives then:

$$
I(h)=\frac{A}{h^{4}}+\sum_{i} B_{i} \cdot \frac{\cos \left(h \delta_{i}+\phi_{1}\right)}{h^{4}}+\ldots \quad, \quad \lim _{h \rightarrow \infty}\left(-\left[h^{3} \cdot I(h)\right]^{\prime} \cdot h \cdot \frac{\sin (h r)}{h r}\right)=\lim _{h \rightarrow \infty}\left(-\frac{\operatorname{const}}{h}\right.
$$

This means that the first term, which arises by the partial integration, vanishes, as it was the case in the first derivative. Thus, after putting in the relation for the first derivative of the correlation function, we get:

$$
\left[r \gamma^{\prime}(r)\right]^{\prime}=r \gamma^{\prime \prime}(r)+\gamma^{\prime}(r)=\frac{1}{r} \int_{0}^{\infty}\left[3 h^{3} I(h)+h^{4} I^{\prime}(h)\right]^{\prime} \cdot \frac{\sin (h r)}{h r} d h
$$

and thus

$$
r \cdot \gamma^{\prime \prime}(r)=\frac{1}{r} \int_{0}^{\infty}\left[3 h^{3} I(h)+h^{4} I^{\prime}(h)\right]^{\prime} \cdot \frac{\sin (h r)}{h r} d h+\frac{1}{r} \int_{0}^{\infty}\left[h^{3} I(h)\right]^{\prime} \cdot \frac{\sin (h r)}{h r} d h .
$$

After writing the right side in one integral and add the derivatives, we obtain:

$$
r \gamma^{\prime \prime}(r)=\frac{1}{r} \int_{0}^{\infty}\left[4 h^{3} I(h)+h^{4} I^{\prime}(h)\right]^{\prime} \cdot \frac{\sin (h r)}{h r} d h \text {. }
$$

So the identity for $0<r$ is proved. Numerous variances of other partial integrations lead to more and more new formulae. With all limiting values POROD's law of SAS must be used in the considerations. This proves that such transformation cannot be successful in all particle systems.

After this special example we have to put the question of a system of further similar possibilities of transformations. In table 1 . the following abbreviations are used:

$$
v(h, r)=\frac{\partial}{\partial h} \frac{\sin (h r)}{h r}, p(h, r)=\frac{\sin (h r)}{h r}, u(h, r)=\int_{0}^{h} \frac{\sin (h r)}{h r} d h, b(h, r)=\int_{0}^{h} J_{0}(h \cdot r) d h
$$

\section{References}

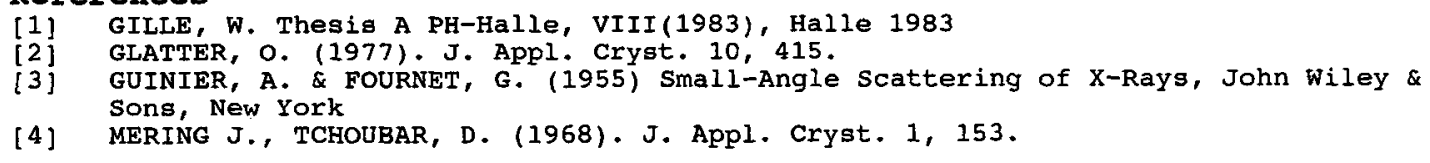

